\title{
New solutions of the heat equation and application to thin plate heat conduction
}

\author{
JACOB MANALE \\ University of South Africa \\ Department of Mathematical Sciences \\ Preller street 32, Pretoria \\ South Africa \\ manaljm@unisa.ac.za
}

\begin{abstract}
Using a Lie symmetry group generator and a generalised form of Euler's formula for solving second order ordinary differential equations, we determine new symmetries for the heat equation, leading to new solutions. As an application, we test a formula resulting from this approach on thin plate heat conduction.
\end{abstract}

Key-Words: Symmetry analysis, Partial differential equations, heat equations.

\section{Introduction}

There is something odd and rarely commented upon that is at the core of some, if not all known analytical solutions of the heat equation

$$
C \frac{\partial^{2} u}{\partial x^{2}}=c \rho \frac{\partial u}{\partial \tau}
$$

or

$$
u_{x x}=u_{t}
$$

where $C$ is known as the thermal conductivity, $c$ the specific heat, $\rho$ the density, $u$ is temperature that depends on position $x$ and time $\tau$, with $t=k \tau$ and $k=C /(c \rho)$, called the thermal diffusivity. The unsettling matter is that the solutions suggest that when $t=0$ we should always have $u=0$, which we find impractical. Impractical in that one then cannot have arbitrary initial conditions like $u\left(t=0, x_{0}\right)=u_{0}$ without $u_{0}=0$. Numerical techniques, on the other hand, do not have this problem, implying that there is some unknown analytical solution waiting to be unearthed.

This we address using Lie's symmetry group theoretical method, a technique he introduced centuries ago through the now famous paper [1]. It is worth noting that this technique has been applied before on this equation, and that two very distinct solutions have consistently resulted. They are in the linear combination

$$
u=\frac{1}{\sqrt{t}} e^{-\frac{x^{2}}{4 t}}\left(C_{1}+C_{2} \frac{x}{t}\right),
$$

and can be found in Bluman's work and the work he did with others, including with Kumei [2]. The same results are also contained in the work with Cole, communicated by Keller [3], also with Anco [4]. The parameter $n$ contained in the result Ibragimov [5] got

$$
u=\frac{C_{0}}{\sqrt{t^{n}}} e^{-\frac{x^{2}}{4 t}},
$$

generates a broader perspective to one of Bluman's solutions. Olsen [6], used a very clever trick to get

$$
u=\frac{x}{2 \sqrt{t}} e^{\frac{-x^{2}}{4 t}} .
$$

Clearly, this result together with (3) and (4) are obviously in the family of solutions of the form

$$
u=f(t, x) e^{-\frac{x^{2}}{4 t}} .
$$

The factor $\exp \left(-x^{2} /(4 t)\right)$ guarantees that $u=0$ when $t=0$.

Other contributions in the field are by Kallianpur, Karandikar [7], Kwok [7], Hui [8], Longstaff [9] and Platen [10]. There are also studies by Naicker, Andriopoulos and Leach [11], Pooe, Mahomed and Soh [12], Sinkala, Leach and OHara [13], and Gazizov and Ibragimov [14].

In this contribution, we determine new symmetries for (2), leading to new invariant solutions. This requires introducing a new infinitesimal parameter $\omega$. Infinitesimal parameters are not new to Symmetry analysis. What we do differently is that the parameter is not introduced into the symmetry generator as is customary, but outside of it. To realise this, we invoke Euler's formulas for solving second order ordinary differential equations, discussed in Appendix A. 
We show that in addition to the family of solutions espoused in (6), there is a whole new family mapped by a paired couple presented in (126). And also suggest (129) as an alternative to averting the arbitrary zero in $u$ when $t=0$.

\section{Solution of the determining equa- tion}

In order to generate point symmetries for equation (2), we first consider a change of variables from $t, x$ and $u$ to $t^{*}, x^{*}$ and $u^{*}$ involving an infinitesimal parameter $\epsilon$. A Taylor's series expansion in $\epsilon$ near $\epsilon=0$ yields

$$
\left.\begin{array}{l}
t^{*} \approx t+\epsilon T(t, x, u) \\
x^{*} \approx x+\epsilon \xi(t, x, u) \\
\left.u^{*} \approx u+\epsilon \zeta t, x, u\right)
\end{array}\right\}
$$

where

$$
\left.\begin{array}{rl}
\left.\frac{\partial t^{*}}{\partial \epsilon}\right|_{\epsilon=0} & =T(t, x, u) \\
\left.\frac{\partial x^{*}}{\partial \epsilon}\right|_{\epsilon=0} & =\xi(t, x, u) \\
\left.\frac{\partial u^{*}}{\partial \epsilon}\right|_{\epsilon=0} & =\quad \zeta t, x, u)
\end{array}\right\} .
$$

The tangent vector field (8) is associated with an operator

$$
X=T \frac{\partial}{\partial t}+\xi \frac{\partial}{\partial x}+\zeta \frac{\partial}{\partial u},
$$

called a symmetry generator. This in turn leads to the invariance condition

$$
\left.X^{[2]}\left(u_{x x}-u_{t}\right)\right|_{\left\{u_{x x}=u_{t}\right\}}=0,
$$

where $X^{[2]}$ is the second prolongation of $X$. It is obtained from the formulas:

$$
\begin{gathered}
X^{[2]}=X+\zeta_{t}^{(1)} \frac{\partial}{\partial u_{t}}+\zeta_{x}^{(1)} \frac{\partial}{\partial u_{x}}+\zeta_{t t}^{(2)} \frac{\partial}{\partial u_{t t}} \\
+\zeta_{t x}^{(2)} \frac{\partial}{\partial u_{t x}}+\zeta_{x x}^{(2)} \frac{\partial}{\partial u_{x x}},
\end{gathered}
$$

where

$$
\begin{gathered}
\zeta_{t}^{(1)}=\frac{\partial g}{\partial t}+u \frac{\partial f}{\partial t}+\left[f-\frac{\partial T}{\partial t}\right] u_{t}-\frac{\partial \xi}{\partial x} u_{x} \\
\zeta_{x}^{(1)}=\frac{\partial g}{\partial x}+u \frac{\partial f}{\partial x}+\left[f-\frac{\partial \xi}{\partial x}\right] u_{x}-\frac{\partial T}{\partial t} u_{t} \\
\zeta_{t t}^{(2)}=\frac{\partial^{2} g}{\partial t^{2}}+u \frac{\partial^{2} f}{\partial t^{2}}+\left[2 \frac{\partial f}{\partial t}-\frac{\partial^{2} T}{\partial t^{2}}\right] u_{t}-\frac{\partial^{2} \xi}{\partial t^{2}} u_{x} \\
+\left[f-2 \frac{\partial T}{\partial t}\right] u_{t t}-2 \frac{\partial \xi}{\partial t} u_{t x}
\end{gathered}
$$

and

$$
\begin{aligned}
& \zeta_{t x}^{(2)}=\frac{\partial^{2} g}{\partial t \partial x}+u \frac{\partial^{2} f}{\partial t \partial x}+\left[2 \frac{\partial f}{\partial x}-\frac{\partial^{2} T}{\partial t \partial x}\right] u_{t} \\
& +\left[2 \frac{\partial f}{\partial t}-\frac{\partial^{2} \xi}{\partial t \partial x}\right] u_{x}-\left[f-\frac{\partial T}{\partial t}-\frac{\partial \xi}{\partial x}\right] u_{t x} \\
& -\frac{\partial T}{\partial x} u_{t t}-\frac{\partial \xi}{\partial t} u_{x x} .
\end{aligned}
$$

It is to be understood here that the simplification $\zeta(t, x, u)=u f(t, x)+g(t, x)$ is adopted from the calculations that led to the old symmetries:

$$
\begin{array}{rlc}
Y_{1}= & \frac{\partial}{\partial x}, \\
Y_{2}= & \frac{\partial}{\partial t}, \\
Y_{3}= & x \frac{\partial}{\partial x}+2 t \frac{\partial}{\partial t}, \\
Y_{4}= & x t \frac{\partial}{\partial x}+t^{2} \frac{\partial}{\partial t}+\left(\frac{t}{2}+\frac{x^{2}}{u}\right) u \frac{\partial}{\partial u}, \\
Y_{5}= & t \frac{\partial}{\partial x}-\frac{x u}{2} \frac{\partial}{\partial u}, \\
Y_{6}= & u \frac{\partial}{\partial u}, \\
Y_{\infty}= & g(t, x) \frac{\partial}{\partial u} .
\end{array}
$$

These are mentioned here to ease comparison with our own, which are at the end of this section.

The invariance condition (10) then leads to the equation

$\frac{\partial^{2} g}{\partial x^{2}}+u \frac{\partial^{2} f}{\partial x^{2}}+\left[2 \frac{\partial f}{\partial x}-\frac{\partial^{2} \xi}{\partial x^{2}}\right] u_{x}-\frac{\partial^{2} T}{\partial x^{2}} u_{t}+$ $\left[f-2 \frac{\partial T}{\partial x}\right] u_{t}-2 \frac{\partial T}{\partial x} u_{t x}-\frac{\partial g}{\partial t}-u \frac{\partial f}{\partial t}-\left[f-\frac{\partial T}{\partial t}\right] u_{t}+$ $\frac{\partial \xi}{\partial x} u_{x}=0$,

called determining equation, from which follows the monomials

$$
\left.\begin{array}{ccc}
u_{t x}: & T_{x}=0 \\
u_{t} & : & T_{t}-2 \xi_{x}=0 \\
u_{x}: & 2 f_{x}-\xi_{x x}+\xi_{t}=0 \\
u & : & f_{x x}-f_{t}=0 \\
1 & : & g_{x x}-g_{t}=0
\end{array}\right\}
$$

called the defining equations.

To begin solving these, we note that the first defining equation $T_{x}=0$, suggests that $T$ should not depend on $x$. The implication is that we would end with less number of symmetries if we continue this way. Fortunately, we now have formula (135) to remedy this.

We use the formula to generate some dependence on $x$ for $T$. That is, $T$ depends on both $t$ and $x$ near $\epsilon=0$, but not at $\epsilon=0$. Differentiating this defining equation with respect to $t$, gives

$$
T_{t x}=0 .
$$

This can then be used to simplify the second defining equation. When the latter is differentiated with respect to $x$, we get

$$
T_{x t}-2 \xi_{x x}=0 .
$$


Because the function $T$ is analytic everywhere, Euler's mixed derivatives theorem holds, meaning $T_{x t}=$ $T_{t x}$. This then reduces (20) into

$$
\xi_{x x}=0,
$$

which then integrates into

$$
\xi=a+x b,
$$

where $a=a(t)$ and $b=b(t)$. This expression is similar to the one appearing with Euler's formulas in the system (131). The discussion there was that the middle expression cannot be transformed into the other two, then we had to introduce (135) to make it possible. That expression becomes handy here, because we can now express (22) in the form

$$
\xi=\frac{a \phi \cos (\omega x / i)+b \sin (\omega x / i)}{\omega / i},
$$

where $\phi=\sin (\omega / i)$. It is clear that (22) reduces to (21) when $\omega \rightarrow 0$. The second defining equation, $T_{t}-$ $2 \xi_{x}=0$, then leads to

$$
T=\frac{-2 \dot{a} \phi \sin (\omega x / i)+2 \dot{b} \cos (\omega x / i)}{\omega}+A_{0},
$$

where $A_{0}$ is a constant. Thus $T$ now appears to also depend on $x$, but we know this is subject to $\omega=0$. Substituting $\xi$ and $T$ from equations (23) and (24) into the third defining equation, $2 f_{x}=\eta_{x x}-\eta_{t}$, leads to

$$
\begin{aligned}
2 f_{x}= & -\frac{\dot{a} \phi}{i} \frac{w}{i} \cos (\omega x / i)-\dot{b} \frac{\omega}{i} \sin (\omega x / i) \\
& -\ddot{a} \frac{\phi}{\omega} \cos (\omega x / i)-\frac{i \ddot{b}}{\omega} \sin (\omega x / i),
\end{aligned}
$$

Integrating this with respect to $x$ gives

$$
\begin{aligned}
f= & -(\dot{a}+\ddot{a}) \frac{\phi}{2} \sin (\omega x / i) \\
& +(\dot{b}-\ddot{b}) \frac{1}{2} \cos (\omega x / i)+\frac{B_{0}}{2},
\end{aligned}
$$

where $B_{0}$ is a constant. We now substitute this into the fourth defining equation to establish the functions $a$ and $b$. First we differentiate (26) once with respect to $t$ :

$$
\begin{aligned}
f_{t}= & -\left(\ddot{a}+a^{(3)}\right) \frac{\phi}{2} \sin (\omega x / i) \\
& +\left(\ddot{b}-b^{(3)}\right) \frac{1}{2} \cos (\omega x / i),
\end{aligned}
$$

then twice with respect to $x$ :

$$
\begin{aligned}
f_{x x}= & -(\dot{a}+\ddot{a}) \frac{\phi}{2} \omega^{2} \sin (\omega x / i) \\
& +(\dot{b}-\ddot{b}) \frac{\omega^{2}}{2} \cos (\omega x / i) .
\end{aligned}
$$

The substitution leads to

$$
(\dot{a}+\ddot{a}) \omega^{2}=\ddot{a}+a^{(3)},
$$

and

$$
(\dot{b}-\ddot{b}) \omega^{2}=\ddot{b}-b^{(3)}
$$

To solve (29), we note it can be written in the form

$$
\frac{\ddot{a}+a^{(3)}}{\dot{a}+\ddot{a}}=\omega^{2} .
$$

That is,

$$
\dot{a}+\ddot{a}=C_{0} e^{\omega^{2} t} .
$$

Subsequently,

$$
a=\frac{C_{0}}{\omega^{2}} \frac{1}{\omega^{2}+1} e^{\omega^{2} t}+C_{1}+C_{2} e^{-t} .
$$

Similarly, solving equation (30) yields

$$
b=\frac{D_{0}}{\omega^{2}} \frac{1}{\omega^{2}-1} e^{\omega^{2} t}+D_{1}+D_{2} e^{t},
$$

for some constants $C_{0}, C_{1}, C_{2}, D_{0}, D_{1}$ and $D_{2}$.

\subsection{Infinitesimals}

The linearly independent solutions of the defining equations (18) lead to the infinitesimals

$$
\begin{aligned}
T= & -2 \phi\left(\frac{C_{0}}{\omega^{4}\left(\omega^{2}+1\right)} e^{\omega^{2} t}\right) \sin (\omega x / i) \\
& -2 \phi\left(C_{1} t-C_{2} e^{-t}\right) \sin (\omega x / i) \\
& +2\left(\frac{D_{0}}{\omega^{4}\left(\omega^{2}-1\right)} e^{\omega^{2} t}\right) \cos (\omega x / i) \\
& +2\left(D_{1} t+D_{2} e^{t}\right) \cos (\omega x / i)+A_{0}, \\
\xi= & \frac{i \phi}{\omega}\left(\frac{C_{0}}{\omega^{2}} \frac{1}{\omega^{2}+1} e^{\omega^{2} t}\right) \cos (\omega x / i) \\
& +\frac{i \phi}{\omega}\left(C_{1}+C_{2} e^{-t}\right) \cos (\omega x / i) \\
& +\frac{i}{\omega}\left(\frac{D_{0}}{\omega^{2}} \frac{1}{\omega^{2}-1} e^{\omega^{2} t}\right) \sin (\omega x / i) \\
& +\frac{i}{\omega}\left(D_{1}+D_{2} e^{t}\right) \sin (\omega x / i)
\end{aligned}
$$

and

$$
\begin{aligned}
f= & -C_{0} \frac{\phi e^{\omega^{2} t}}{2} \sin (\omega x / i) \\
& -D_{0} \frac{e^{\omega^{2} t}}{2} \cos (\omega x / i)+\frac{B_{0}}{2} .
\end{aligned}
$$




\subsection{The symmetries}

According to (9), the infinitesimals: (35), (36) and (37), lead to the generators

$$
\begin{aligned}
X_{1}= & \frac{2 e^{\omega^{2} t}}{\omega^{4}\left(\omega^{2}-1\right)} \cos (\omega x / i) \frac{\partial}{\partial t} \\
+ & \frac{i e^{\omega^{2} t}}{\omega^{3}\left(\omega^{2}-1\right)} \sin (\omega x / i) \frac{\partial}{\partial x} \\
- & \frac{e^{\omega^{2} t}}{2} \cos (\omega x / i) u \frac{\partial}{\partial u}, \\
X_{2}= & -\frac{2 \phi e^{\omega^{2} t}}{\omega^{4}\left(\omega^{2}+1\right)} \sin (\omega x / i) \frac{\partial}{\partial t} \\
+ & \frac{i \phi e^{\omega^{2} t}}{\omega^{3}\left(\omega^{2}+1\right)} \cos (\omega x / i) \frac{\partial}{\partial x} \\
-\quad & \frac{\phi e^{\omega^{2} t}}{2} \sin (\omega x / i) u \frac{\partial}{\partial u}, \\
X_{3}= & -2 \phi t \sin (\omega x / i) \frac{\partial}{\partial t} \\
& +\frac{i \phi}{\omega} \cos (\omega x / i) \frac{\partial}{\partial x}, \\
X_{6}= & 2 e^{t} \cos (\omega x / i) \frac{\partial}{\partial t} \\
& +\frac{i}{\omega} e^{t} \sin (\omega x / i) \frac{\partial}{\partial x}, \\
X_{5}= & 2 \phi e^{-t} \sin (\omega x / i) \frac{\partial}{\partial t} \\
X_{4}= & 2 t \cos (\omega x / i) \frac{\partial}{\partial t} \\
& +\frac{i}{\omega} \sin (\omega x / i) \frac{\partial}{\partial x}
\end{aligned}
$$

The last defining equation leads to an infinite symmetry generator.

$$
X_{\infty}=g(t, x) \frac{\partial}{\partial u}
$$

\section{Construction of invariant solutions for (2)}

The symmetries $X_{7}, X_{8}$ and $X_{\infty}$ are not different from $Y_{2}, Y_{6}$ and $Y_{\infty}$ obtained by Bluman and others, as such unlikely to lead to anything not already known. We limit our construction of invariant solutions to $X_{1}$ and $X_{2}$, as they appear to be broader and more encompassing than $X_{3}, X_{4}, X_{5}$, and $X_{6}$. What is certain is that $X_{3}$ and $X_{4}$ are automatically addressed.

\subsection{Invariant solutions through the symme- try $X_{1}$}

The characteristic equations that arise from the symmetry $X_{1}$ :

$$
\begin{aligned}
\frac{\omega^{4}\left(\omega^{2}-1\right) e^{-\omega^{2} t} d t}{2 \cos (\omega x / i)}= & \frac{i \omega^{3}\left(\omega^{2}-1\right) e^{-\omega^{2} t} d x}{\sin (\omega x / i)} \\
& =\frac{2 e^{-\omega^{2} t} d u}{\cos (\omega x / i) u}
\end{aligned}
$$

lead to

$$
\frac{\omega^{4}\left(\omega^{2}-1\right) e^{-\omega^{2} t} d t}{\cos (\omega x / i)}=2 \frac{i \omega^{3}\left(\omega^{2}-1\right) e^{-\omega^{2} t} d x}{\sin (\omega x / i)},
$$

and

$$
\frac{\omega^{4}\left(\omega^{2}-1\right) e^{-\omega^{2} t} d t}{2 \cos (\omega x / i)}=\frac{2 e^{-\omega^{2} t} d u}{\cos (\omega x / i) u} .
$$

Equation (48) becomes

$$
\omega^{2} d t=-2 \frac{(\omega / i) \cos (\omega x / i) d x}{\sin (\omega x / i)},
$$

so that

$$
\lambda=-\omega^{2} t-2 \ln |\sin (\omega x / i)| .
$$

Hence,

$$
\eta=e^{\frac{\omega^{2}}{2} t}|\sin (\omega x / i)|
$$

where $\eta=\exp (-\lambda / 2)$.

Equation (49) becomes

$$
\frac{\omega^{4}\left(\omega^{2}-1\right) d t}{4}=\frac{d u}{u},
$$

so that the invariant solution has the form

$$
u=e^{\left(\omega^{4}\left(\omega^{2}-1\right)\right) t / 4} \phi(\eta) .
$$


This means

$$
\begin{aligned}
u_{t}= & \frac{\omega^{4}\left(\omega^{2}-1\right)}{4} e^{\left(\omega^{4}\left(\omega^{2}-1\right)\right) t / 4} \phi \\
& +e^{\left(\omega^{4}\left(\omega^{2}-1\right)\right) t / 4} \dot{\phi} \eta_{t} .
\end{aligned}
$$

That is,

$$
\begin{aligned}
u_{t}= & \frac{\omega^{4}\left(\omega^{2}-1\right)}{4} e^{\left(\omega^{4}\left(\omega^{2}-1\right)\right) t / 4} \phi \\
& +\frac{\omega^{2}}{2} \eta e^{\left(\omega^{4}\left(\omega^{2}-1\right)\right) t / 4} \dot{\phi} .
\end{aligned}
$$

On the other hand,

$$
u_{x}=e^{\left(\omega^{4}\left(\omega^{2}-1\right)\right) t / 4} \dot{\phi} \eta_{x},
$$

so that

$$
\begin{aligned}
u_{x x}= & e^{\left(\omega^{4}\left(\omega^{2}-1\right)\right) t / 4} \ddot{\phi}\left(\eta_{x}\right)^{2} \\
& +e^{\left(\omega^{4}\left(\omega^{2}-1\right)\right) t / 4} \dot{\phi} \eta_{x x} .
\end{aligned}
$$

That is,

$$
\begin{aligned}
& u_{x x}=e^{\left(\omega^{4}\left(\omega^{2}-1\right)\right) t / 4} \ddot{\phi} \\
& \times\left( \pm e^{\frac{\omega^{2}}{2} t}(-\omega / i) \frac{\cos (\omega x / i)}{\omega}\right)^{2} \\
& -e^{\left(\omega^{4}\left(\omega^{2}-1\right)\right) t / 4} \dot{\phi} \\
& \times\left(\mp e^{-\frac{\omega^{2}}{2} t}(-\omega / i)^{2} \frac{\sin (\omega x / i)}{\omega}\right),
\end{aligned}
$$

or

$$
\begin{aligned}
u_{x x}= & \omega^{2} e^{\left(\omega^{4}\left(\omega^{2}-1\right)\right) t / 4} \ddot{\phi}\left(e^{\omega^{2} t}-\eta^{2}\right) \\
& +\omega^{2} \eta e^{\left(\omega^{4}\left(\omega^{2}-1\right)\right) t / 4} \dot{\phi} .
\end{aligned}
$$

Substituting the expression for $u_{t}$ from equation (56) and the one for $u_{x x}$ from equation (60) into (2), give

$$
\begin{aligned}
& \omega^{2} \ddot{\phi}\left(e^{\omega^{2} t}-\eta^{2}\right)+\omega^{2} \eta \dot{\phi} \\
& =\frac{\omega^{4}\left(\omega^{2}-1\right)}{4} \phi+\frac{\omega^{2}}{2} \eta \dot{\phi} .
\end{aligned}
$$

In the limit $\omega$ approaching zero, this equation reduces to

$$
\left(1-\eta^{2}\right) \ddot{\phi}+\frac{\eta}{2} \dot{\phi}=0 .
$$

That is,

$$
\frac{\ddot{\phi}}{\dot{\phi}}=\frac{1}{2} \frac{\eta}{\eta^{2}-1},
$$

so that

$$
\int_{\eta_{1}}^{\eta_{2}} \frac{d}{d \eta}(\ln \dot{\phi}) d \eta=\frac{1}{2} \int_{\eta_{1}}^{\eta_{2}} \frac{\tilde{\eta}}{\tilde{\eta}^{2}-1} d \tilde{\eta} .
$$

The integral on the left evaluates easily. Hence,

$$
\ln \dot{\phi}=\tilde{F}_{0}+\frac{1}{2} \int_{\eta_{1}}^{\eta_{2}} \frac{\tilde{\eta}}{\tilde{\eta}^{2}-1} d \tilde{\eta},
$$

where $\tilde{F}_{0}$ is a constant. The other requires letting $\eta_{1}=$ $\eta$ and $\eta_{2}=\eta+\omega$ then invoking L'hopital's principle. That is,

$$
\ln \dot{\phi}=\tilde{F}_{0}+\frac{\frac{\omega}{2} \frac{d \eta}{d \omega} \frac{d}{d \eta} \int_{\eta}^{\eta+\omega} \frac{\tilde{\eta}}{\tilde{\eta}^{2}-1} d \tilde{\eta}}{\frac{d}{d \omega} \omega}
$$

Evaluating $d \eta / d \omega$ :

$$
\begin{aligned}
& \ln \dot{\phi}=\tilde{F}_{0}+ \\
& \frac{\omega}{2}\left(\frac{\omega}{2} t|\sin (\omega x / i)| \pm(x / i) \cos (\omega x / i)\right) \\
& \times e^{\frac{\omega^{2}}{2}} t \frac{d}{d \eta} \int_{\eta}^{\eta+\omega} \frac{\tilde{\eta}}{\tilde{\eta}^{2}-1} d \tilde{\eta} .
\end{aligned}
$$

The fundamental theorem of calculus ensures that the derivative removes the integral, simplifying the equation to

$$
\begin{aligned}
& \ln \dot{\phi}=\tilde{F}_{0}+ \\
& \frac{\omega}{2}\left(e^{\frac{\omega^{2}}{2} t}|\sin (\omega x / i)| \pm(x / i) \cos (\omega x / i)\right) \\
& \times e^{\frac{\omega^{2}}{2} t} \frac{\eta}{\eta^{2}-1} .
\end{aligned}
$$

A further simplification on the right gives

$$
\begin{aligned}
& \ln \dot{\phi}=\tilde{F}_{0}+ \\
& \frac{\omega}{2}\left(\frac{\omega}{2} t|\sin (\omega x / i)| \pm(x / i) \cos (\omega x / i)\right) \\
& \times \frac{\omega e^{\frac{\omega^{2}}{2} t} \frac{|\sin (\omega x / i)|}{\omega}}{\eta^{2} e^{-\frac{\omega^{2}}{2} t}-e^{-\frac{\omega^{2}}{2} t}} .
\end{aligned}
$$

That is,

$$
\begin{aligned}
& \ln \dot{\phi}=\tilde{F}_{0}+ \\
& \frac{\omega^{2}}{2}\left(\frac{\omega}{2} t|\sin (\omega x / i)| \pm(x / i) \cos (\omega x / i)\right) \\
& \times \frac{e^{\frac{\omega^{2}}{2} t}( \pm x / i) \cos (\omega x / i)}{\eta^{2} e^{-\frac{\omega^{2}}{2} t}-e^{-\frac{\omega^{2}}{2} t}}
\end{aligned}
$$


so that

$$
\begin{aligned}
& \ln \dot{\phi}=\tilde{F}_{0}+ \\
& \frac{\frac{\omega^{2}}{2}\left(\frac{\omega}{2} t|\sin (\omega x / i)| \pm(x / i) \cos (\omega x / i)\right)}{|\sin (\omega x / i)|^{2} e^{\frac{\omega^{2}}{2} t}-e^{-\frac{\omega^{2}}{2} t}} \\
& \times e^{\frac{\omega^{2}}{2} t}( \pm x / i) \cos (\omega x / i) .
\end{aligned}
$$

That is,

$$
\begin{aligned}
& \ln \dot{\phi}=\tilde{F}_{0}+ \\
& \frac{\frac{\omega^{2}}{2}\left(\frac{\omega}{2} t|\sin (\omega x / i)| \pm(x / i) \cos (\omega x / i)\right)}{(-\cos (\omega x / i))^{2} e^{\frac{\omega^{2}}{2} t}+e^{\frac{\omega^{2}}{2} t}-e^{-\frac{\omega^{2}}{2} t}} \\
& \times e^{\frac{\omega^{2}}{2} t}( \pm x / i) \cos (\omega x / i) .
\end{aligned}
$$

The trigonometric and hyperbolic identities ensure that there are further simplifications in the denominator. Hence,

$$
\begin{aligned}
& \ln \dot{\phi}=\tilde{F}_{0}+ \\
& \frac{\frac{\omega^{2}}{2}\left(\frac{\omega}{2} t|\sin (\omega x / i)| \pm(x / i) \cos (\omega x / i)\right)}{(-\cos (\omega x / i))^{2} e^{\frac{\omega^{2}}{2} t}+2 i \sin \left(\frac{\omega^{2}}{2 i} t\right)} \\
& \times e^{\frac{\omega^{2}}{2} t}( \pm x / i) \cos (\omega x / i) .
\end{aligned}
$$

Evaluating the limits:

$$
\ln \dot{\phi}=\tilde{F}_{0}+\frac{-x^{2}}{4 t} .
$$

That is,

$$
\dot{\phi}=F_{0} e^{\frac{-x^{2}}{4 t}},
$$

with $F_{0}=\exp \left(\tilde{F}_{0}\right)$. Hence,

$$
\phi=F_{0} \int_{\eta_{1}}^{\eta_{2}} e^{\frac{-x^{2}}{4 t}} d \tilde{\eta} .
$$

The solutions for (2) follows from (54). The above expression then leads to

$$
u=e^{\left(\omega^{4}\left(\omega^{2}-1\right)\right) t / 4}\left[F_{0} \int_{\eta_{1}}^{\eta_{2}} e^{\frac{-x^{2}}{4 t}} d \tilde{\eta}\right] .
$$

\subsubsection{The first solution through $X_{1}$}

When $F_{0}=-i A / \omega$ and $\omega=0$ inside the integral in (77), we get

$$
u=A e^{\left(\omega^{4}\left(\omega^{2}-1\right)\right) t / 4} \int_{x_{1}}^{x_{2}} e^{\frac{-x^{2}}{4 t}} d x .
$$

The plot of this result is given in Figure 1. What is in Figure 2 is the same solution obtained through other means by Fassari and Rinaldi [15].

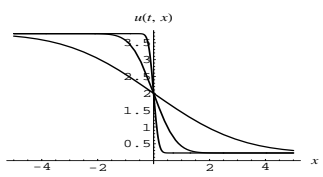

Figure 1: Plot of the solution in (78) for equation (2).

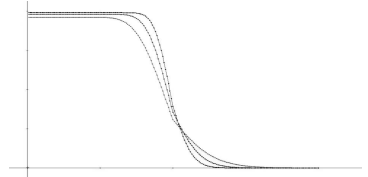

Figure 2: Plot of the solution obtained by Fassari and Rinaldi [15] for equation (2), similar to the one in Figure 1 .

\subsubsection{The second solutions through $X_{1}$ : Olsen's result.}

The second solution for (2) follows from a slight modification of invariant $\phi$ 's coefficient $e^{\left(\omega^{4}\left(\omega^{2}-1\right)\right) t / 4}$ in (76). It is replaced by the expression developed in Appendix B, given in (144). Hence,

$$
u=\frac{1}{\sqrt{\left(\omega^{2}-1\right) t}} \frac{F_{0} \int_{\eta_{1}}^{\eta_{2}} e^{\frac{-x^{2}}{4 t}} d \tilde{\eta}}{\omega^{2}} .
$$

This then invokes L'hopital's principle in the limit $\omega$ going to zero with $\eta_{1}=\eta$ and $\eta_{2}=\eta+\omega$. That is,

$$
u=\frac{1}{\sqrt{\left(\omega^{2}-1\right) t}} \frac{\frac{d \eta}{d \omega} \frac{d}{d \eta} F_{0} \int_{\eta}^{\eta+\omega} e^{\frac{-x^{2}}{4 t}} d \tilde{\eta}}{2 \omega} .
$$

That is,

$$
u=\frac{1}{\sqrt{\left(\omega^{2}-1\right) t}} \frac{\frac{d \eta}{d \omega} \frac{d}{d \eta} F_{0} \int_{\eta}^{\eta+\omega} e^{\frac{-x^{2}}{4 t}} d \tilde{\eta}}{2 \omega} .
$$

Hence,

$$
u=F_{0} \frac{x}{2 \sqrt{t}} e^{\frac{-x^{2}}{4 t}} .
$$

This solution is sketched in Figure 3. A similar result by Richards and Abrahamsen [16] is in Figure 4. 


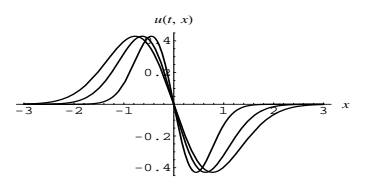

Figure 3: Plot of the solution in (82) for equation (2).

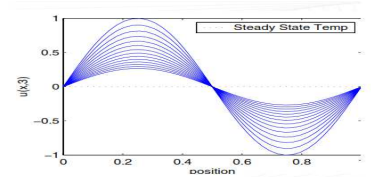

Figure 4: Plot of the solution obtained by Richards and Abrahamsen [16] for equation (2), similar to the one in Figure 3.

\subsubsection{The third solution through $X_{1}$ : Bluman's result.}

Another solution is possible out of (81), and is made possible by The factor $e^{\omega^{2} t / 2}$ in $\eta$ with $\mu=$ $\omega^{2} t / 2$. It takes the form

$$
u=F_{0} \frac{x}{2 t^{3 / 2}} e^{\frac{-x^{2}}{4 t}} .
$$

This result is the same as the second component in Bluman's solution with $C_{2}=F_{0} / 2$.

\subsection{Invariant solutions through the symme- try $X_{2}$}

Determining solutions through $X_{2}$ is very much the same as through $X_{1}$, because the two symmetries are very much alike. The invariants have similar forms. That is,

$$
\eta=e^{\frac{\omega^{2}}{2} t}|\cos (\omega x / i)|
$$

and

$$
u=e^{\left(\omega^{4}\left(\omega^{2}+1\right)\right) t / 4} \phi(\eta)
$$

This means

$$
\begin{aligned}
u_{t}= & \frac{\omega^{4}\left(\omega^{2}+1\right)}{4} e^{\left(\omega^{4}\left(\omega^{2}+1\right)\right) t / 4} \phi \\
& +e^{\left(\omega^{4}\left(\omega^{2}+1\right)\right) t / 4} \dot{\phi} \eta_{t} .
\end{aligned}
$$

That is,

$$
\begin{aligned}
u_{t}= & \frac{\omega^{4}\left(\omega^{2}+1\right)}{4} e^{\left(\omega^{4}\left(\omega^{2}+1\right)\right) t / 4} \phi \\
& +\frac{\omega^{2}}{2} \eta e^{\left(\omega^{4}\left(\omega^{2}+1\right)\right) t / 4} \dot{\phi} .
\end{aligned}
$$

On the other hand,

$$
u_{x}=e^{\left(\omega^{4}\left(\omega^{2}+1\right)\right) t / 4} \dot{\phi} \eta_{x}
$$

so that

$$
\begin{aligned}
u_{x x}= & e^{\left(\omega^{4}\left(\omega^{2}+1\right)\right) t / 4} \ddot{\phi}\left(\eta_{x}\right)^{2} \\
& +e^{\left(\omega^{4}\left(\omega^{2}+1\right)\right) t / 4} \dot{\phi} \eta_{x x} .
\end{aligned}
$$

That is,

$$
\begin{aligned}
& u_{x x}=e^{\left(\omega^{4}\left(\omega^{2}+1\right)\right) t / 4} \ddot{\phi} \\
& \times\left(-e^{-\frac{\omega^{2}}{2} t}(-\omega / i) \frac{\sin (\omega x / i)}{\omega}\right)^{2} \\
& -e^{\left(\omega^{4}\left(\omega^{2}+1\right)\right) t / 4} \dot{\phi} \\
& \times\left(e^{-\frac{\omega^{2}}{2} t}(-\omega / i)^{2} \frac{\cos (\omega x / i)}{\omega}\right),
\end{aligned}
$$

or

$$
\begin{aligned}
u_{x x}= & \omega^{2} e^{\left(\omega^{4}\left(\omega^{2}+1\right)\right) t / 4} \ddot{\phi}\left(e^{-\omega^{2} t}-\eta^{2}\right) \\
& +\omega^{2} \eta e^{\left(\omega^{4}\left(\omega^{2}+1\right)\right) t / 4} \dot{\phi} .
\end{aligned}
$$

Substituting the expression for $u_{t}$ from equation (87) and the one for $u_{x x}$ from equation (91) into (2), give

$$
\begin{aligned}
& \omega^{2} \ddot{\phi}\left(e^{-\omega^{2} t}-\eta^{2}\right)+\omega^{2} \eta \dot{\phi} \\
& =\frac{\omega^{4}\left(\omega^{2}+1\right)}{4} \phi+\frac{\omega^{2}}{2} \eta \dot{\phi} .
\end{aligned}
$$

In the limit $\omega$ approaching zero, this equation reduces to

$$
\left(1-\eta^{2}\right) \ddot{\phi}+\frac{\eta}{2} \dot{\phi}=0
$$

That is,

$$
\frac{\ddot{\phi}}{\dot{\phi}}=\frac{1}{2} \frac{\eta}{\eta^{2}-1}
$$

so that

$$
\int \frac{d}{d \eta}(\ln \dot{\phi}) d \eta=F_{0}+\frac{1}{2} \int \frac{\eta}{\eta^{2}-1} d \eta,
$$

where $F_{0}$ is a constant. 
The Gaussian function solution follows from reversing the integral on the right side of equation (95). This requires the use of L'hopital's principle. We induce this by introducing $\omega$ to the denominator, and another one in the numerator for balance:

$$
\int \frac{d}{d \eta}(\ln \dot{\phi}) d \eta=\omega \frac{\frac{1}{2} \int \frac{\eta}{\eta^{2}-1} d \eta}{\omega} .
$$

Next, we use the result

$$
\lim _{\omega \rightarrow 0}\left[\int_{\eta}^{\eta+\omega} \frac{\tilde{\eta}}{\tilde{\eta}^{2}-1} d \tilde{\eta}\right]=0,
$$

in conjunction with L'hopital's principle on (96), to yield

$$
\int \frac{d}{d \eta}(\ln \dot{\phi}) d \eta=\omega \frac{\frac{1}{2} \frac{d}{d \omega} \int \frac{\eta}{\eta^{2}-1} d \eta}{\frac{d \omega}{d \omega}},
$$

so that

$$
\int \frac{d}{d \eta}(\ln \dot{\phi}) d \eta=\frac{\omega}{2} \frac{d \eta}{d \omega} \frac{d}{d \eta} \int \frac{\eta}{\eta^{2}-1} d \eta .
$$

Now introducing the value for $\eta$ to the coefficient:

$$
\begin{aligned}
& \int \frac{d}{d \eta}(\ln \dot{\phi}) d \eta \\
& =\frac{\omega}{2}(\omega|\cos (\omega x / i)|-( \pm x / i) \sin (\omega x / i)) \\
& \times e^{\frac{\omega^{2}}{2} t} \frac{d}{d \eta} \int \frac{\eta}{\eta^{2}-1} d \eta .
\end{aligned}
$$

The fundamental theorem of calculus ensures that the derivative removes the integral, simplifying the equation to

$$
\begin{aligned}
& \int \frac{d}{d \eta}(\ln \dot{\phi}) d \eta \\
& =\frac{\omega}{2}(\omega|\cos (\omega x / i)|-( \pm x / i) \sin (\omega x / i)) \\
& \times e^{\frac{\omega^{2}}{2} t} \frac{\eta}{\eta^{2}-1}
\end{aligned}
$$

A further simplification on the right gives

$$
\begin{aligned}
& \int \frac{d}{d \eta}(\ln \dot{\phi}) d \eta \\
& =\frac{\omega}{2}(\omega t|\cos (\omega x / i)|-( \pm x / i) \sin (\omega x / i)) \\
& \times \frac{|\cos (\omega x / i)|}{\eta^{2} e^{-\frac{\omega^{2}}{2} t}-e^{-\frac{\omega^{2}}{2} t}}
\end{aligned}
$$

Since $\omega$ is small, we get

$$
\begin{aligned}
& \int \frac{d}{d \eta}(\ln \dot{\phi}) d \eta \\
& =\frac{\omega}{2}(\omega t|\cos (\omega x / i)|-( \pm x / i) \sin (\omega x / i)) \\
& \times \frac{|\cos (\omega x / i)|}{\eta^{2} e^{-\frac{\omega^{2}}{2} t}-e^{-\frac{\omega^{2}}{2} t}}
\end{aligned}
$$

so that

$$
\begin{aligned}
& \int \frac{d}{d \eta}(\ln \dot{\phi}) d \eta \\
& =\frac{\frac{\omega}{2}(\omega t|\cos (\omega x / i)|-( \pm x / i) \sin (\omega x / i))}{(\cos (\omega x / i))^{2} e^{\frac{\omega^{2}}{2} t}-e^{-\frac{\omega^{2}}{2} t}} \\
& \times|\cos (\omega x / i)| .
\end{aligned}
$$

That is,

$$
\begin{aligned}
& \int \frac{d}{d \eta}(\ln \dot{\phi}) d \eta \\
& =\frac{\frac{\omega}{2}(\omega t|\cos (\omega x / i)|-( \pm x / i) \sin (\omega x / i))}{(-\sin (\omega x / i))^{2} e^{\frac{\omega^{2}}{2} t}+e^{\frac{\omega^{2}}{2} t}-e^{-\frac{\omega^{2}}{2} t}} \\
& \times|\cos (\omega x / i)| .
\end{aligned}
$$

The first solution is

$$
\begin{aligned}
& \int \frac{d}{d \eta}(\ln \dot{\phi}) d \eta \\
& =\frac{\frac{\omega^{2}}{2}\left(t|\cos (\omega x / i)|-( \pm x / i) \frac{\sin (\omega x / i)}{\omega}\right)}{(-\sin (\omega x / i))^{2} e^{\frac{\omega^{2}}{2} t}+2 i \sin \left(\frac{\omega^{2}}{2 i} t\right)} \\
& \times|\cos (\omega x / i)|,
\end{aligned}
$$

so that

$$
\int \frac{d}{d \eta}(\ln \dot{\phi}) d \eta=-\frac{x^{2}}{4 t} .
$$

That is,

$$
\dot{\phi}=F_{0} e^{\frac{-x^{2}}{4 t}},
$$

or

$$
\phi=F_{1}+F_{0} \int e^{\frac{-x^{2}}{4 t}} d \eta,
$$

where $F_{0}$ is a constant. The expression for $u$ then assumes the form

$$
u=e^{\left(\omega^{4}\left(\omega^{2}-1\right)\right) t / 4}\left[F_{1}+F_{0} \int e^{\frac{-x^{2}}{4 t}} d \eta\right] .
$$

\subsubsection{The first solution through $X_{2}$}

When $\omega=0$ and $F_{0}=-A / \omega$ in (110), we get

$$
u=\left[F_{1}+F_{0} \int e^{\frac{-x^{2}}{4 t}} x d x\right],
$$

so that

$$
u=F_{1}+A e^{\frac{-x^{2}}{4 t}} .
$$

This solution is plotted in Figure 5. 


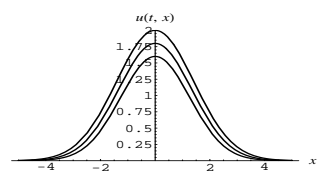

Figure 5: Plot of the solution in (112) for equation (2).

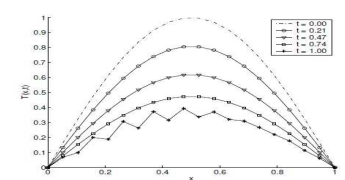

Figure 6: Plot of the solution by Gerald Recktenwald, similar to the one in Figure 5.

\subsubsection{The second solution through $X_{2}$ : Bluman's second result.}

The second solution through $X_{2}$ follows a similar procedure as was for $X_{1}$, leading to

$$
u=\frac{A}{2 \sqrt{t}} e^{\frac{-x^{2}}{4 t}} .
$$

This result is the same as the first component in Bluman's solution with $C_{1}=1 / 2$. It is sketched in Figure 7. A similar result by Balluffi, Allen and Carter [17] is in Figure 8.

\subsubsection{The third solution through $X_{2}$ : Ibragi- mov's result.} form

Like the second solution, a third solution takes the

$$
u=\frac{A}{2 t^{3 / 2}} e^{\frac{-x^{2}}{4 t}} .
$$

This result is the same is a special case of Ibragimov's solution with $n=3$.

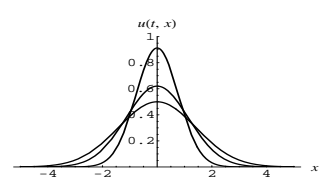

Figure 7: Plot of the solution in (113) for equation (2).

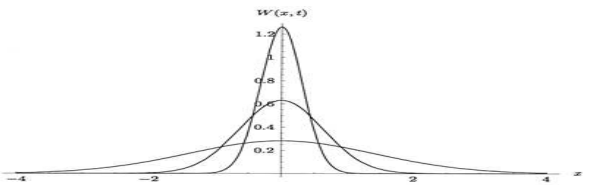

Figure 8: Plot of the solution obtained by Balluffi, Allen and Carter [17] for equation (2), similar to the one in Figure 7.

\section{Other solutions through $X_{2}$}

More solutions follow from evaluating the limits in (101) by following a different path, leading to

$$
\begin{aligned}
& \int \frac{d}{d \eta}(\ln \dot{\phi}) d \eta \\
& =\frac{\frac{\omega^{2}}{2}\{-( \pm x / i)(x / i)\}}{(-\sin (\omega x / i))^{2} e^{\frac{\omega^{2}}{2} t}+2 i \sin \left(\frac{\omega^{2}}{2 i} t\right)} \\
& \times \cos (\omega x / i)|\cos (\omega x / i)|
\end{aligned}
$$

so that

$$
\int \frac{d}{d \eta}(\ln \dot{\phi}) d \eta=\mp \frac{x^{2}}{2\left(x^{2}-t^{2}\right)} .
$$

Hence,

$$
u=F_{1}+e^{\omega^{4}\left(\omega^{2}+1\right)} F_{0} \int_{\eta_{1}}^{\eta_{2}} e^{ \pm \frac{x^{2}}{2\left(x^{2}-t^{2}\right)}} d \tilde{\eta} .
$$

\subsubsection{A first couple of solutions through $X_{2}$}

A simple pair of solutions results from (117) when $\omega$ goes to zero requires. That is,

$$
u=F_{1}+F_{0} \int_{\eta_{1}}^{\eta_{2}} e^{-\frac{x^{2}}{2\left(x^{2}-t^{2}\right)}} d \tilde{\eta}
$$

and

$$
u=F_{1}+F_{0} \int_{\eta_{1}}^{\eta_{2}} e^{\frac{x^{2}}{2\left(x^{2}-t^{2}\right)}} d \tilde{\eta}
$$

\subsubsection{A second couple of solutions through $X_{2}$}

Setting $\eta_{1}=\eta$ and $\eta_{2}=\eta+\omega$ in (117) and letting $\omega$ go to zero requires that $F_{0}=-A / \omega$ for some constant $A$. This invokes L'hopital's principle, so that

$$
u=F_{1}+A e^{-\frac{x^{2}}{2\left(x^{2}-t^{2}\right)}}
$$

and

$$
u=F_{1}+A e^{\frac{x^{2}}{2\left(x^{2}-t^{2}\right)}} .
$$




\subsubsection{A third couple of solutions through $X_{2}$}

As was the case for $X_{1}$, the limits in Appendix B can be used to create more solutions. The following pair results:

$$
u=F_{1}+\frac{A}{\sqrt{t}} e^{-\frac{x^{2}}{2\left(x^{2}-t^{2}\right)}}
$$

and

$$
u=F_{1}+\frac{A}{\sqrt{t}} e^{\frac{x^{2}}{2\left(x^{2}-t^{2}\right)}} .
$$

\subsubsection{A fourth couple of solutions through $X_{2}$}

Continuing with the argument started in the preceding section leads to the fourth couple of solutions:

$$
u=F_{1}+\frac{A}{t^{3 / 2}} e^{-\frac{x^{2}}{2\left(x^{2}-t^{2}\right)}}
$$

and

$$
u=F_{1}+\frac{A}{t^{3 / 2}} e^{\frac{x^{2}}{2\left(x^{2}-t^{2}\right)}} .
$$

It is apparent from these calculations that though $X_{2}$ are largely of the family

$$
u=f(t, x) e^{ \pm \frac{x^{2}}{2\left(x^{2}-t^{2}\right)}} .
$$

\section{Applications: Heat conduction in thin plates.}

As mentioned in the Introduction, there are many methods used in practice to solve (2), an equation that finds application in a number of different situations. The backward heat equation

$$
u_{x x}=-u_{t},
$$

too, does arise in practice. Unfortunately, without analytical solutions, one could end up applying one of the two equations to a situation to which it does not apply.

For example, in a study on heat conduction in thin plates, Hancork [18] deduced solutions for (2) presented in Figure 9. These we unpack in Figures 10, 11 and 12 using (120). Unfortunately, practical results indicate it is (127) which is applicable to this situation. This we deduce from the fact that impractical singularities arise when $u$ is plotted against $t$ when (120) is used, but disappear when this expression assumes the form

$$
u=F_{1}+A e^{-\frac{x^{2}}{2\left(x^{2}+t^{2}\right)}},
$$

satisfying both (127) and empirical results, plotted in Figure 13 . These are clearly in the family of the form

$$
u=f(t, x) e^{ \pm \frac{x^{2}}{2\left(x^{2}+t^{2}\right)}}
$$

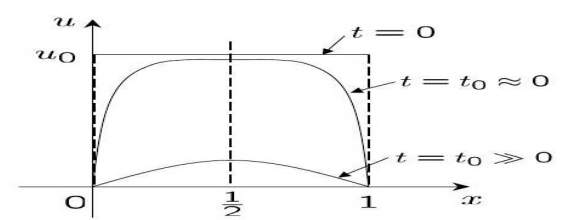

Figure 9: Plot of the solution obtained by Hancork [18] for equation (2) for cases $t=0, t \approx 0$ and $t>>$ 0 , all stacked onto the same sketch.

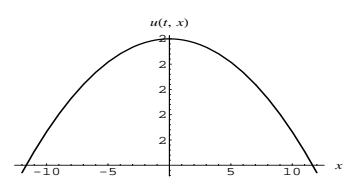

Figure 10: Plot of the solution in (120) for equation (2), similar to the one in Figure 9 for $t=t_{0}>>0$.

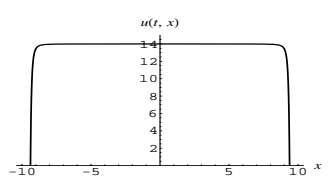

Figure 11: Plot of the solution in (120) for equation (2), similar to the one in Figure 9 for $t=t_{0} \approx 0$.

\section{Conclusion}

In this study, new symmetries were obtained for the heat equation, and two were used to determine group 


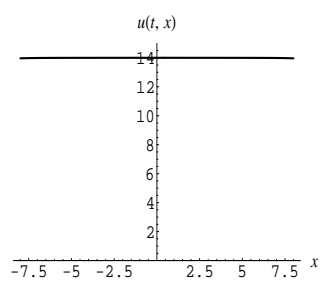

Figure 12: Plot of the solution in (120) for equation (2), similar to the one in Figure 9 for $t=t_{0}=0$.

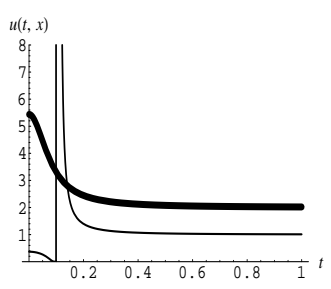

Figure 13: The solid curve is from (128) for the backward heat equation (127), while the other curves are from (120) for the heat equation (2).

invariant solutions. It was shown they do not only lead to solutions possible through old symmetries, but also to new solutions, including the ones possible through other methods.

\section{APPENDIX A: Generalising Euler's formu- las for solving second order ordinary differential equations}

It is well-known that Lie's group theoretical methods seek to reduce procedures for solving differential equations of any challenging form to simple ones that may also have the form

$$
a_{0} \ddot{y}+b_{0} \dot{y}+c_{0} y=0,
$$

for $y=y(x)$, with parameters $a_{0}, b_{0}$ and $c_{0}$. It is also that accepted Euler's formulas are suitable for solving

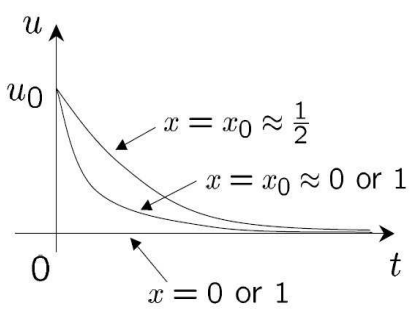

Figure 14: The temperature against time curve obtained by Hancork, valid for $t \geq 1 / \pi^{2}$, comparable to the solution in Figure 13.

such equations. They are:

$$
y=\left\{\begin{array}{cc}
e^{-\frac{b_{0}}{2 a_{0}} x}\left(A e^{-\tilde{\omega} x}+B e^{\tilde{\omega} x}\right), & b_{0}^{2}>4 a_{0} c_{0}, \\
A+B x, & b_{0}^{2}=4 a_{0} c_{0}, \\
e^{-\frac{b_{0}}{2 a_{0}} x}[A \cos (\tilde{\omega} x)] & \\
+B e^{-\frac{b_{0}}{2 a_{0}} x}[\sin (\tilde{\omega} x)], & b_{0}^{2}<4 a_{0} c_{0}
\end{array}\right.
$$

where $\tilde{\omega}=\sqrt{b_{0}^{2}-4 a_{0} c_{0}} /\left(2 a_{0}\right)$.

But there is a problem with this system: It does not reduce to $y=A+B x$ when $b_{0}=c_{0}=0$. This is because Euler did not solve the equation to get the formulas. There has never been a need to do so, primarily because the formulas have been very successful in applications, and they still are.

The need for an exact solution here, is driven by the desire understand solutions for equation (2) through symmetry methods. It is impossible through Euler's formulas. To get such exact formula, first let

$$
y=\beta z,
$$

with $\beta=\beta(x)$ and $z=z(x)$, so that

$$
\dot{y}=\dot{\beta} z+\beta \dot{z},
$$

and

$$
\ddot{y}=\ddot{\beta} z+2 \dot{\beta} \dot{z}+\beta \ddot{z} .
$$

These transform (130) into

$$
a_{0}(\ddot{\beta} z+2 \dot{\beta} \dot{z}+\beta \ddot{z})+b_{0}(\dot{\beta} z+\beta \dot{z})+c_{0} \beta z=0 .
$$

That is,

$a_{0} \beta \ddot{z}+\left(2 a_{0} \dot{\beta}+b_{0} \beta\right) \dot{z}+\left(a_{0} \ddot{\beta}+b_{0} \dot{\beta}+c_{0} \beta\right) z=0$. 
Choosing $\beta$ to satisfy $2 a_{0} \dot{\beta}+b_{0} \beta=0$ simplifies equation (132). That is,

$$
\beta=C_{00} e^{\frac{-b_{0}}{2 a_{0}} x},
$$

for some constant $C_{00}$. Equation (132) assumes the form

$$
\ddot{z}=-\frac{a_{0} \ddot{\beta}+b_{0} \dot{\beta}+c_{0} \beta}{a_{0} \beta} z .
$$

That is,

$$
\ddot{z}=\left(\frac{b_{0}^{2}-4 a_{0} c_{0}}{4 a_{0}^{2}}\right) z .
$$

But $\ddot{z}$ can be written as $\dot{z} d z / d x$. Therefore,

$$
\dot{z} \frac{d \dot{z}}{d z}=\left(\frac{b_{0}^{2}-4 a_{0} c_{0}}{4 a_{0}^{2}}\right) z,
$$

or

$$
\dot{z} d \dot{z}=\left(\frac{b_{0}^{2}-4 a_{0} c_{0}}{4 a_{0}^{2}}\right) z d z .
$$

That is,

$$
\frac{\dot{z}^{2}}{2}=\left(\frac{b_{0}^{2}-4 a_{0} c_{0}}{4 a_{0}^{2}}\right) \frac{z^{2}}{2}+C_{01},
$$

for some constant $C_{01}$. That is,

$$
\dot{z}=\sqrt{\left(\frac{b_{0}^{2}-4 a_{0} c_{0}}{4 a_{0}^{2}}\right) \frac{z^{2}}{2}+C_{01}},
$$

or

$$
\frac{d z}{\sqrt{\left(\frac{b_{0}^{2}-4 a_{0} c_{0}}{4 a_{0}^{2}}\right) z^{2}+2 C_{01}}}=d x
$$

That is,

$$
\frac{d z}{\sqrt{A_{00}^{2}-z^{2}}}=\sqrt{-\frac{b_{0}^{2}-4 a_{0} c_{0}}{4 a_{0}^{2}}} d x,
$$

with $A_{00}^{2}=2 C_{01} / \sqrt{-\frac{b_{0}^{2}-4 a_{0} c_{0}}{4 a_{0}^{2}}}$. Hence,

$$
\begin{aligned}
z= & \frac{2 C_{01}}{\sqrt{-\frac{b_{0}^{2}-4 a_{0} c_{0}}{4 a_{0}^{2}}}} \\
& \times \sin \left(\sqrt{-\frac{b_{0}^{2}-4 a_{0} c_{0}}{4 a_{0}^{2}}} x+C_{02}\right)
\end{aligned}
$$

for some constant $C_{02}$. That is,

$$
\begin{aligned}
y= & C_{00} e^{\frac{-b_{0}}{2 a_{0}} x} \frac{2 C_{01}}{\sqrt{-\frac{b_{0}^{2}-4 a_{0} c_{0}}{4 a_{0}^{2}}}} \\
& \times \sin \left(\sqrt{-\frac{b_{0}^{2}-4 a_{0} c_{0}}{4 a_{0}^{2}}} x+C_{02}\right)
\end{aligned}
$$

Letting

$$
\bar{\omega}=\sqrt{-\frac{b_{0}^{2}-4 a_{0} c_{0}}{4 a_{0}^{2}}}
$$

we have

$$
y=C_{00} e^{\frac{-b_{0}}{2 a_{0}} x} \frac{2 C_{01}}{\bar{\omega}} \sin \left(\bar{\omega} x+C_{02}\right),
$$

or

$y=C_{00} e^{\frac{-b_{0}}{2 a_{0}} x} \quad 2 C_{01} \quad\left[\frac{\sin \left(C_{02}\right)}{\bar{\omega}} \cos (\bar{\omega} x)+\right.$ $\left.\cos \left(C_{02}\right) \frac{\sin (\bar{\omega} x)}{\bar{\omega}}\right]$.

A reduction to the trivial case $\ddot{y}=0$ requires that $\sin \left(C_{02}\right)=C_{03} \sin (\bar{\omega})$ and $\cos \left(C_{02}\right)=C_{04} \cos (\bar{\omega})$. That is, $C_{03}^{2}+C_{04}^{2}=1$. Hence,

$y=C_{00} e^{\frac{-b_{0}}{2 a_{0}} x} \quad 2 C_{01} \quad\left[\frac{C_{03} \sin (\bar{\omega})}{\bar{\omega}} \cos (\bar{\omega} x)+\right.$ $\left.C_{04} \cos (\bar{\omega}) \frac{\sin (\bar{\omega} x)}{\bar{\omega}}\right]$,

or simply

$$
\begin{aligned}
y= & C_{00} e^{\frac{-b_{0}}{2 a_{0}} x} 2 C_{01} \frac{C_{03} \sin (\bar{\omega}) \cos (\bar{\omega} x)}{\bar{\omega}} \\
& +C_{00} e^{\frac{-b_{0}}{2 a_{0}} x} 2 C_{01} \frac{C_{04} \sin (\bar{\omega} x)}{\bar{\omega}} .
\end{aligned}
$$

It is very vital to indicate that if the parameters $\bar{\omega}$ in the denominator and $\sin (\bar{\omega})$ are absorbed into the coefficients $C_{01}$ and $C_{03}$, then formula (135) would reduce to one of Euler's formulas. But the consequences would be fatal, as formula (135) would not reduce to $y=A+B x$ when $b_{0}=c_{0}=0$, that is, when $\bar{\omega}=0$.

Unfortunately, this result cannot be found in any university textbook.

\section{APPENDIX B: Useful limit results}

It is true that

$$
\lim _{\mu \rightarrow 0}\left\{\frac{\sin \left(\frac{\mu t}{i}\right)}{\mu}\right\}=\frac{t}{i} .
$$

This can be written in the form

$$
\lim _{\mu \rightarrow 0}\left\{\frac{\sin \left(\frac{\mu x}{i}\right)}{\mu}-\frac{t}{i}\right\}=0,
$$

or

$$
\lim _{\mu \rightarrow 0}\left\{\frac{\sin \left(\frac{\mu t}{i}\right)}{\mu}-\frac{t}{i} \cos \left(\frac{\mu t}{i}\right)\right\}=0 .
$$

Removing the 'lim' for greater clarity:

$$
\frac{\sin \left(\frac{\mu t}{i}\right)}{\mu}=\frac{t}{i} \cos \left(\frac{\mu t}{i}\right) \text {. }
$$


That is,

$$
\sin \left(\frac{\mu t}{i}\right)=\frac{t}{i} \mu \cos \left(\frac{\mu t}{i}\right)
$$

or

$$
\cos \left(\frac{\mu t}{i}\right)=\frac{i}{t} \frac{\sin \left(\frac{\mu t}{i}\right)}{\mu} .
$$

We then have

$$
\frac{\cos \left(\frac{\mu t}{i}\right)}{\mu^{q}}=\mu \frac{\cos \left(\frac{\mu t}{i}\right)}{\mu^{q+1}} .
$$

Carrying out the derivative on the right hand side:

$$
\frac{\cos \left(\frac{\mu t}{i}\right)}{\mu^{q}}=\frac{-\mu\left(\frac{t}{i}\right) \sin \left(\frac{\mu t}{i}\right)+\cos \left(\frac{\mu t}{i}\right)}{\mu^{q+1}} .
$$

Substituting (136):

$$
\frac{\cos \left(\frac{\mu t}{i}\right)}{\mu^{q}}=\frac{-\mu^{2}\left(\frac{t}{i}\right)^{2} \cos \left(\frac{\mu t}{i}\right)+\cos \left(\frac{\mu t}{i}\right)}{\mu^{q+1}} .
$$

That is,

$$
\mu \cos \left(\frac{\mu t}{i}\right)=\mu^{2} t^{2} \cos \left(\frac{\mu t}{i}\right)+\cos \left(\frac{\mu t}{i}\right),
$$

which can be expressed in the form

$$
\mu^{2} \cos \left(\frac{\mu t}{i}\right)-\mu^{3} t^{2} \cos \left(\frac{\mu t}{i}\right)=\frac{i}{t} \sin \left(\frac{\mu t}{i}\right) .
$$

Since $\sin \left(\frac{\mu t}{i}\right)=0$ for $\mu$ small, it follows then that

$$
\mu^{2} \cos \left(\frac{\mu t}{i}\right)=\mu^{3} t^{2} \cos \left(\frac{\mu t}{i}\right) .
$$

Since $e^{\mu t}$ ca be expressed in the form $\cos (\mu t / i)+$ $i \sin (\mu t / i)$, then

$$
\mu^{2} e^{\mu t}=\mu^{3} t^{2} \cos \left(\frac{\mu t}{i}\right)
$$

so that

$$
\sqrt{\mu} e^{\mu t / 4}=\left[\mu^{3} \cos \left(\frac{\mu t}{i}\right)\right]^{\frac{1}{4}} \sqrt{t},
$$

or

$$
\sqrt{\mu} e^{-\mu t / 4}=\left[\mu^{3} \cos \left(\frac{\mu t}{i}\right)\right]^{\frac{1}{4}} \sqrt{t},
$$

Therefore (54) and (54) can then be written in the form

$$
u=\frac{\sqrt{\mu}}{\left[\mu^{3} \cos \left(\frac{\mu t}{i}\right)\right]^{\frac{1}{4}} \sqrt{t}} \phi(\eta),
$$

with $\mu=\omega^{4}\left(\omega^{2}-1\right)$ in the case of (54) and $\mu=$ $\omega^{4}\left(\omega^{2}+1\right)$ for $(85)$. That is,

$$
u=\frac{1}{\sqrt{\left(\omega^{2}-1\right) t} \omega^{2}} \phi(\eta)
$$

for (54), and

$$
u=\frac{1}{\sqrt{\left(\omega^{2}+1\right) t} \omega^{2}} \phi(\eta)
$$

for (85).

\section{References:}

[1] Lie S. On integration of a class of linear partial differential equations by means of definite integrals. Arch. Math., 3:328-368, 1881.

[2] Bluman G. W. and Kumei S. Symmetries and Differential Equations, volume 81. SpringerVerlag, New York, 1989.

[3] Keller J. B. The general similarity solution of the heat equation. J. Math. Mech., 18:1025-1042, 1969.

[4] Bluman G. W. and Anco S. C. Symmetry and Integration Methods for Differential Equations, volume 154. Springer-Verlag, New York, 2000.

[5] N.I. Ibragimov. Selected works: Lie group analysis, differential equations, riemannian geometry, lie backlund groups, mathematical physics. Grad. Texts in Math., I, 2006.

[6] Olsen H. H. Similarity solutions for the heat equation. PDE EBOOK, 2004.

[7] Kwok Y. K. Mathematical Models of Financial Derivatives. Springer-Verlag, Singapore, 1998.

[8] Lo C. F. and Hui C. H. Valuation of financial derivatives with time-independent parameters: Lie-algebraic approach. Quantitative Finance, 1:73-78, 2001.

[9] Longstaff F. A. A nonlinear general equilibrium model of the term structure of interest rates. $J$. Financ. Econ., 23:195-224, 1989.

[10] Platen E. A minimal financial market model, in: M. kohlmann, s. tang (eds.), mathematical finance. pages 293-301, 2001.

[11] Andriopoulos K. Naicker V. and Leach P. G. L. Symmetry reductions of a hamiltonjacobibellman equation arisingin financial mathematics. $J$. Nonlinear Math. Phys., 12 (2):268-283, 2005. 
[12] Mahomed F. M. Pooe C. A. and Soh C. W. Fundamental solutions for zero-coupon bond pricing models. Nonlinear Dynam., 36:69-76, 2004.

[13] Leach P. G. L. Sinkala W. and OHara J. G. Zerocoupon bond prices in the vasicek and cir models: Their computationas group-invariant solutions. Math. Methods Appl. Sci., 31:665-678, 2008.

[14] Gazizov R. K. and Ibragimov N. H. Lie symmetry analysis of differential equations in finance. Nonlinear Dynam., 17 (4):387-407, 1998.

[15] Fassari S. and Rinaldi F. On some potential applications of the heat equation with a repulsive point interaction to derivative pricing. Rendiconti di Matematica, 31:35-52, 2011.

[16] American Society of Mechanical Engineers. Journal of Heat Transfer. Number v. 124, nos. 3-4 in Transactions of the ASME. American Society of Mechanical Engineers, 2002.

[17] Allen S. M. Balluffi R. W. and Carter W. C. Kinetics of Materials. Wiley and Sons, 2005.

[18] Hancock M. J. and Bush J. W. M. Evaporative instabilities in climbing films. J. Fluid Mech., 466:285-304, 2002.

\section{Creative Commons Attribution License 4.0 (Attribution 4.0 International, CC BY 4.0)}

This article is published under the terms of the Creative Commons Attribution License 4.0

https://creativecommons.org/licenses/by/4.0/deed.en_US 